\title{
Evaluación del desempeño del sistema de gestión de JUDC en FAREM Matagalpa
}

\author{
Elmer Miroslav Mosher Golovin \\ Tutora: MSc. Natalia Golovina
}

\section{RESUMEN}

El presente trabajo consiste en evaluar el desempeño del sistema para la gestión de la Jornada Universitaria de Desarrollo Científico (JUDC) en la FAREM Matagalpa de acuerdo con estándares ISO 9126 y CMMI, durante el 2014. La intención de esta investigación es la verificación del software y capacidades del recurso humano mediante las herramientas ISO 9126 y CMMI, lo que permitirá detectar e identificar los diferentes problemas y fallas al introducir datos al sistema y utilizarlos, posibilitando así la implementación de mejoras para garantizar la adecuación y calidad del sistema informático. Se utilizarán diversas técnicas para la recolección de información: observación no participativa a toda la población, entrevistas a profundidad a coordinación de investigación, grupos focales a jurados y alumnos ayudantes, estudios de caso tanto individuales como grupales y evaluaciones al sistema informático. Respecto a las deficiencias que se encuentren en la evaluación inicial CMMI no se realizarán correcciones debido a los costos y el poco personal disponible para capacitaciones, pero en relación a ISO 9126 se realizarán correcciones exhaustivas en todos los aspectos del software. Luego de las correcciones se elaborará una evaluación final ISO 9126 y CMMI. Los principales problemas se detectarán tanto en la fase de llenado de datos, como en la fase de manejo del sistema. El trabajo servirá para culminar la implementación exitosa del sistema para agilizar y hacer más confiable el proceso de evaluación de trabajos de JUDC.

\section{ABSTRACT}

The present work consists on evaluating the performance of the system for management of the Universitary Journey of Scientific Development (JUDC by its Spanish acronym) in the FAREM Matagalpa according to ISO 9126 and CMMI standards, during 2014. The purpose of this research is the verification of the software and human resource capabilities using the ISO 9126 and CMMI tools, allowing the detection and identification of the different problems and failures when introducing and using data to the system, enabling this way the implementation of improvements to guarantee the adequacy and quality of the informatic system. Different techniques to collect information were used: non participative observation to all the population, deep interviews to the research coordination, focus groups to jurors and student assistants, individual and grupal case studies and evaluations to the informatic system. According to the deficiencies found in the initial CMMI evaluation corrections were not made due to the costs and few personnel available for capacitations, but regarding to ISO 9126 exhaustive corrections will be made in all the aspects of the software. After the corrections a final ISO 9126 and CMMI evaluation will be made. The principal problems will be detected both in the data filling phase, as the system using phase. The work will serve to complete the successful implementation of the system to streamline and make more reliable the process of evaluating works of the JUDC. 


\section{INTRODUCCIÓN}

Actualmente la utilización de soluciones informáticas se ha vuelto una necesidad en las organizaciones, pues brinda tantas ventajas competitivas e institucionales que la organización que no utiliza tales soluciones se ve obligada a retirarse del mercado. Desde la década del noventa ha surgido la necesidad de software de calidad, pues durante el auge informático eran tantos los desarrolladores de software y la competencia que la calidad era un factor muy descuidado.

Para dar respuesta a las fallas en la calidad del software se originan los equipos dedicados en exclusiva al control del desarrollo, apoyándose de la opinión experta de organizaciones como ISO (International Standarization Organization, en español Organización Internacional para la Estandarización).

Existen dos evaluaciones fundamentales para garantizar el mejor rendimiento de un producto software: la evaluación al mismo producto software, y la evaluación al usuario del software. Mientras que la primera evaluación se enfoca en detalles muy técnicos de la solución informática, la segunda evaluación se enfoca en determinar las habilidades del usuario y como éstas afectarán la productividad cuando se utilice el software.

El objeto de estudio de ésta investigación se centra en evaluar el desempeño del sistema de gestión de JUDC en FAREM Matagalpa de acuerdo con estándares ISO 9126 y CMMI, período 2014.

Para ello se realizará una evaluación ISO 9126 y CMMI inicial a diferentes procesos y elementos fundamentales del sistema de gestión de JUDC. De los resultados que se obtengan se profundizará en los aspectos con mayores fallas para así tener información clara y pertinente para la mejora del sistema informático.

El presente documento se estructura en base a los objetivos específicos planteados, estando las variables de estudio respaldadas por un marco teórico que brinda un soporte científico a la investigación. La metodología a utilizar se basa en la información expuesta en el diseño metodológico, en la cual se plasma el tipo y enfoque de investigación, población y muestra, técnicas de recopilación de datos, instrumentos y variables de estudio.

\section{ANTECEDENTES}

Evaluar sistemas informáticos es un tópico del cual poco se aborda a nivel mundial, pues se confía mucho en que basta con una buena documentación y requisitos bien definidos, más sin 
embargo existen elementos como la portabilidad o eficiencia los cuales es casi seguro que se descuidan. Las evaluaciones a sistemas informáticos permiten enfocarse en estos elementos descuidados para así dar además una garantía y certificación de calidad al software.

En España, Marcos (2006), realizó un estudio donde evalúa la usabilidad en sistemas de información terminológicos online, encontrándose la utilidad de la ayuda, efectividad y satisfacción como los elementos que más se podrían mejorar, por lo cual el autor recomienda a los responsables de estos sistemas atender los aspectos de usabilidad, pues los usuarios tienen una percepción negativa del contenido de los recursos.

En México, Macedo Ojeda, Vizmanos Lamotte, \& Hunot Alexander (2007), analizan diferentes paquetes informáticos para valoración dietética dirigidos al nutriólogo, en los que se encuentran fallas en la estabilidad, contenido de ayuda, gráficos y maleabilidad y crecimiento de la base de datos, más sin embargo algunos de los paquetes informáticos evaluados no presentan fallas mayores. Los autores sugieren la evaluación previa de las necesidades informáticas del nutriólogo para así seleccionar el software más adecuado.

Se consultó la biblioteca de la UNAN Managua - FAREM Matagalpa para verificar la existencia de bibliografía sobre la evaluación de sistemas informáticos, no encontrándose información al respecto.

\section{JUSTIFICACIÓN}

Esta investigación consiste en evaluar un sistema para manejo y registro de notas de la JUDC en la UNAN Managua - FAREM Matagalpa para determinar las mejoras que pudiese brindar.

Al evaluar el sistema se podrán verificar las diferentes mejoras que ofrezca para los diferentes procesos realizados en la JUDC a través de nuevos y refinados reportes, formularios con mayor funcionalidad y simplicidad, menores tiempos de espera, capacidad para automatizar mayor cantidad de procedimientos, mayor portabilidad del software, entre otros. También esta evaluación permitirá descubrir las fallas que puedan existir en el sistema a evaluar, así como inconsistencias de cálculo, errores de formato, problemas de compatibilidad, fallas de conectividad, entre otros.

El presente estudio por lo tanto beneficiará directamente al área de investigación e indirectamente a docentes y estudiantes de la universidad ya que de esta manera se proporcionará una herramienta de trabajo evaluada, más segura según las necesidades de los usuarios. 
También la investigación servirá como material bibliográfico en el FAREM Matagalpa, y además el autor se enriquecerá con el nuevo conocimiento.

\section{FORMULACIÓN DEL PROBLEMA}

El manejo y registro de notas de la JUDC en la UNAN Managua - FAREM Matagalpa es un proceso que todavía tiene actividades muy iterativas, pues a pesar de contar con un sistema informático, éste no se realizó tomando en cuenta los requerimientos adecuados por lo que varias actividades se realizan manualmente.

Es posible que con un sistema informático adecuado el área de investigación automatice varios de sus procesos, reduciendo tiempo y esfuerzo necesarios para completar la JUDC.

Como pronóstico, si esta situación sigue presentándose, el área de investigación seguirá siendo dependiente de la ayuda de tanto recurso humano, el cual necesita tiempo y esfuerzo de considerable magnitud.

A través de una evaluación a un sistema que cumpla con los requerimientos adecuados, se podrán agilizar los diferentes procesos dando solución a la dificultad existente.

Por tal motivo, se plantea el siguiente problema de investigación:

¿Cómo se desempeña el sistema de gestión de JUDC en la UNAN Managua - FAREM Matagalpa, período 2014?

\section{REFERENCIAS TEÓRICAS}

\section{Particularidades sobre ISO 9126 y CMMI}

\section{Métodos adecuados de evaluación}

Menciona Espelt (1998), que la obtención de información precisa y relevante está relacionada con la existencia de una base de datos adecuada, índices apropiados e interfaces que proporcionen esta información de una manera organizada y sencilla. Es importante que el software no se centre en una parte particular del mismo, pues puede que al esmerarse en dejar con gran atractivo las interfaces se descuide la funcionalidad lógica de las mismas ocasionando una serie de fallas y errores en el software al utilizar tal interfaz. Es vital realizar una buena labor en el diseño de 
la base de datos que utilice un software, pues de ello depende la facilidad de minar datos y obtener información precisa de una manera rápida y ágil, influyendo de manera directa en la calidad del producto software y la experiencia positiva que el usuario pueda tener.

\section{Experiencias}

Johnson, Griffiths, \& Hartley (2003) en su trabajo respecto a un marco de trabajo para evaluación de sistemas de recuperación de información aseguran que la evaluación de software es un procedimiento necesario, pues esto revela a manera precisa los diferentes aspectos negativos que puedan surgir al utilizar tal software. Un elemento importante a tomar en cuenta al utilizar un software nuevo es el nivel de calidad que pueda tener el mismo, pues es diferente utilizar software de Oracle Corporation que se sabe que tuvo que pasar por un proceso de calidad, a utilizar un software "clandestino" del cual no se sabe si ha pasado por un proceso de calidad. Es relativamente común encontrarse con paquetes software del cual se desconoce totalmente la empresa que lo desarrolló, agregando incertidumbre al nivel de calidad que tal software pueda tener. La utilización de estándares de desarrollo y obtención de premios y alianzas permite a la empresa desarrolladora tener un mejor porte, pues esto asegura que el software producto de tal empresa tiene cierto grado de calidad en vez de estar en incertidumbre tal calidad.

\section{Heurísticas}

Acorde a Nielsen (1995b), la evaluación heurística consiste en tener un pequeño grupo de evaluadores que examinen por separado interfaces y juzguen si cumplen con principios de usabilidad reconocidos, intercambiando al final sus resultados de evaluación. Cuando los evaluadores comparten todas sus evaluaciones, se obtienen resultados más fiables y precisos que en la evaluación tradicional. Es muy deseable realizar este tipo de evaluaciones debido a sus niveles de eficacia, más sin embargo lo común es que no hay un equipo evaluador si no que hay un solo individuo evaluador, imposibilitando la evaluación heurística debido a que requiere de varios evaluadores. En la práctica resulta útil apoyarse de un asesor o evaluador ayudante, pues esto permite realizar contrastes entre diferentes puntos de vista hasta llegar a un resultado no preferencista que contiene datos más veraces y precisos. También al tener un compañero evaluador 
se agrega un mayor grado de credibilidad a los resultados, pues ya no se tendrá la mera opinión de alguien, sino que se tendrá el resultado de una discusión entre dos o más expertos.

\section{Patrones de diseño}

Asegura Nielsen (1995a), que existen diez elementos primordiales a tomar en cuenta para los diseños de interfaces, entre los cuales destacan: consistencia y estándares, prevención de errores, flexibilidad y eficiencia de uso, diseño estético y minimalista, y ayuda y documentación. Al desarrollar software por lo general la meta es lograr que simplemente funcione, descuidando una serie de elementos que terminan restando la calidad del software y experiencia positiva del usuario, por lo cual es vital atender a estos elementos apropiadamente. La buena relación entre el usuario final y el software depende de la calidad del software y las habilidades que el usuario tenga para manipular programas informáticos, por ejemplo, un usuario que utiliza un PC por primera vez tropezará en la utilización de un simple procesador de texto, o bien, un usuario experto tendrá problemas al utilizar un software de compleja utilización y que no posea documentación alguna.

\section{Parámetros de evaluación de ISO 9126 y CMMI}

La ISO mide los siguientes criterios/métricas para diferentes factores:

Tabla 1. La medición de diferentes factores según ISO 9126

\begin{tabular}{|l|l|}
\hline \multicolumn{1}{|c|}{ Factor } & \multicolumn{1}{c|}{ Criterios/métricas } \\
\hline Funcional & - Idóneo \\
& - Preciso \\
& - Interoperable \\
& - Seguro \\
\hline Fiable & - Madurez \\
& - Tolerancia a fallos \\
& - Capacidad de recuperación \\
\hline Usable & - Inteligible \\
& - Fácil aprendizaje \\
& - Operable \\
& - Atractivo \\
\hline Eficiente & - Comportamiento en el tiempo \\
& - Utilización de recursos \\
\hline Mantenible & - Analizable \\
& - Cambiable \\
& - Estable \\
& - Probable (de poder realizar pruebas) \\
\hline
\end{tabular}




\begin{tabular}{|c|l|}
\hline \multicolumn{1}{|c|}{ Factor } & \multicolumn{1}{c|}{ Criterios/métricas } \\
\hline Portable & - Adaptable \\
& - Fácil de instalar \\
& - Coexistencia \\
& - Intercambiable \\
\hline Calidad de Uso & - Eficacia \\
& - Productividad \\
& - Satisfacción \\
\hline
\end{tabular}

Fuente: Elaboración propia a partir de parámetros de evaluación y generalidades

El CMMI evalúa diferentes áreas de proceso las cuales se agrupan por categoría y nivel de madurez:

Tabla 2. Áreas de proceso evaluadas por el CMMI

\begin{tabular}{|c|c|c|}
\hline Nivel de madurez & Categoría & Área de proceso \\
\hline \multirow[t]{11}{*}{ Definido } & Gestión de procesos & $\begin{array}{l}\text { Definición de proceso } \\
\text { organizacional }\end{array}$ \\
\hline & & $\begin{array}{l}\text { Enfoque de proceso } \\
\text { organizacional }\end{array}$ \\
\hline & & $\begin{array}{l}\text { Entrenamiento } \\
\text { organizacional }\end{array}$ \\
\hline & & $\begin{array}{l}\text { Dirección integrada de } \\
\text { proyectos }\end{array}$ \\
\hline & & Gestión de riesgos \\
\hline & Ingeniería & Integración del producto \\
\hline & & $\begin{array}{l}\text { Desarrollo de } \\
\text { requerimientos }\end{array}$ \\
\hline & & Solución técnica \\
\hline & & Validación \\
\hline & & Verificación \\
\hline & Apoyo a proyectos & $\begin{array}{l}\text { Análisis de decisiones y } \\
\text { resolución }\end{array}$ \\
\hline \multirow[t]{7}{*}{ Administrado } & Gestión de proyectos & $\begin{array}{l}\text { Monitoreo y control de } \\
\text { proyectos }\end{array}$ \\
\hline & & Planificación de proyectos \\
\hline & & Gestión de requerimientos \\
\hline & & $\begin{array}{l}\text { Administración de } \\
\text { acuerdos con el proveedor }\end{array}$ \\
\hline & Apoyo a proyectos & $\begin{array}{l}\text { Gestión de la } \\
\text { configuración }\end{array}$ \\
\hline & & Medición y análisis \\
\hline & & $\begin{array}{l}\text { Proceso y garantía de } \\
\text { calidad del producto }\end{array}$ \\
\hline Optimizado & Gestión de procesos & Gestión del desempeño \\
\hline
\end{tabular}




\begin{tabular}{|l|l|l|}
\hline & & organizacional \\
\hline & Apoyo a proyectos & $\begin{array}{l}\text { Análisis causal y } \\
\text { resolución }\end{array}$ \\
\hline
\end{tabular}

Fuente: Elaboración propia a partir de parámetros de evaluación y generalidades

\section{PREGUNTAS DIRECTRICES}

- ¿Cómo es el cumplimiento del software y capacidades del recurso humano respecto a las herramientas ISO 9126/CMMI?

- ¿Qué problemas hay en la fase de llenado de datos?

- ¿Cuáles son las fallas en el proceso de manejo del sistema?

\section{DISEÑO METODOLÓGICO}

Se aplicará un enfoque de investigación cualitativo con elementos cuantitativos. El enfoque predominante es cualitativo debido a que la naturaleza del tema prefiere cualidades (evaluación descriptiva), además que la población es pequeña y permite la utilización de instrumentos cualitativos.

Habrá elementos cuantitativos, pues existirá una evaluación inicial ISO 9126 y CMMI que por naturaleza son valoraciones muy numéricas y porcentuales.

Acorde al alcance de la investigación, el presente documento cumple con el tipo de investigación descriptiva, pues se expondrá cómo suceden las cosas, características, comportamientos, etc., tal y como son.

El diseño es no experimental, pues no se manipularán las variables y se limitará a describir los sucesos a como son, sin modificarse. El corte es longitudinal, pues la evaluación a ejecutar no ha empezado y lo que se realizará contemplará toda la evaluación.

El área de estudio es la UNAN Managua - FAREM Matagalpa, pues es donde la JUDC tiene efecto y están todos los individuos de la población. La población está conformada por un jefe del área de investigación, aproximadamente treinta y seis jurados calificadores y unos treinta alumnos ayudantes, sumando un total de sesenta y siete aproximadamente. Por ser pequeña la población, no se extraerá muestra de la misma.

Se utilizarán diversas técnicas para la recolección de información: observación no participativa a toda la población, entrevistas a profundidad con el jefe de investigación, grupos 
focales a jurados y alumnos ayudantes, estudios de caso tanto individuales como grupales a toda la población, y evaluaciones del sistema informático.

Para respaldar la información se utilizará el método teórico que incluye: bibliografía fiable, sintetizar, elementos interactivos claros (cuadros sinópticos, mapas conceptuales, comparativos en tablas, etc.; también conocidos como estrategias), método de redacción (tres niveles), inducir, deducir. También se utilizará el método empírico al apoyarse de la información obtenida de las técnicas de recopilación de datos y experiencia del investigador y población.

Para el análisis de la información se realizará la triangulación, donde la teoría e instrumentos se contrastan y relacionan, revelando así resultados fiables. Para procesar los datos se utilizarán matrices de resultados, software de computadora como SPSS y Microsoft Office Excel, correlación, etc. Para el desarrollo, análisis y elaboración del informe final se usará computadora, impresora, tóner, papel, grabaciones, entre otros.

Las variables a estudiar son: desempeño del sistema, evaluación ISO 9126 al sistema, y evaluación CMMI al entorno.

Por ser variables para un proceso de investigación cualitativa, no se operacionalizan, y su descripción está resumida en la siguiente tabla:

\begin{tabular}{|c|c|c|}
\hline Variable & Sub variable & Descriptor \\
\hline ISO 9126 al sistema & Funcional & Idóneo \\
\hline \multirow{19}{*}{$\begin{array}{l}\text { La ISO } 9126 \text { permite saber el } \\
\text { nivel de calidad que un } \\
\text { sistema tiene. Realizar esta } \\
\text { evaluación permitiría tener un } \\
\text { punto de partida general } \\
\text { sobre cómo se encuentra } \\
\text { actualmente el sistema JUDC. }\end{array}$} & & Preciso \\
\hline & & Interoperable \\
\hline & & Seguro \\
\hline & Confiable & Madurez \\
\hline & & Capacidad de recuperación \\
\hline & & Tolerancia a fallos \\
\hline & Utilizable & Inteligible \\
\hline & & Facilidad de aprendizaje \\
\hline & & Operable \\
\hline & & Atractivo \\
\hline & Portable & Adaptable \\
\hline & & Facilidad de instalación \\
\hline & & Coexistencia \\
\hline & & Intercambiabilidad \\
\hline & Mantenimiento & Analizable \\
\hline & & Modificable \\
\hline & & Estable \\
\hline & & Pruebas \\
\hline & Calidad de uso & Eficacia \\
\hline
\end{tabular}




\begin{tabular}{|c|c|c|}
\hline Variable & Sub variable & Descriptor \\
\hline & & Productividad \\
\hline & & Satisfacción \\
\hline & Eficiencia & Comportamiento en el tiempo \\
\hline & & Utilización de recursos \\
\hline & & Gestión de procesos \\
\hline CMMI al entorno & Definido & Gestión de proyectos \\
\hline \multirow{6}{*}{$\begin{array}{l}\text { El CMMI brinda información } \\
\text { clave sobre el nivel de } \\
\text { habilidades del personal, así } \\
\text { como la calidad de los } \\
\text { recursos con los que se } \\
\text { cuenta. Realizar una } \\
\text { evaluación daría una } \\
\text { percepción clara sobre las } \\
\text { habilidades informáticas del } \\
\text { personal para así aislar } \\
\text { problemas del software y } \\
\text { debilidades del personal }\end{array}$} & & Ingeniería \\
\hline & & Apoyo a proyectos \\
\hline & Administrado & Gestión de proyectos \\
\hline & & Apoyo a proyectos \\
\hline & Optimizado & Gestión de procesos \\
\hline & & Apoyo a proyectos \\
\hline Desempeño del sistema & $\begin{array}{l}\text { Dificultades del } \\
\text { usuario en introducir } \\
\text { datos }\end{array}$ & Malas descripciones \\
\hline \multirow{12}{*}{$\begin{array}{l}\text { En base a las evaluaciones } \\
\text { ISO } 9126 \text { y CMMI, se } \\
\text { evaluarán a mayor detalle los } \\
\text { elementos que mayores } \\
\text { dificultades presenten, para } \\
\text { así obtener un diagnóstico de } \\
\text { mayor calidad }\end{array}$} & & Falta de ayuda \\
\hline & & Formularios incómodos \\
\hline & & $\begin{array}{l}\text { Repetición en la introducción de } \\
\text { datos }\end{array}$ \\
\hline & $\begin{array}{l}\text { Fallas del sistema en } \\
\text { interpretar los datos } \\
\text { que se introduzcan }\end{array}$ & Conversión inadecuada de tipos \\
\hline & & Pérdida de precisión \\
\hline & & No garantía del guardado \\
\hline & $\begin{array}{l}\text { Dificultades del } \\
\text { usuario en manejar el } \\
\text { sistema }\end{array}$ & Controles inadecuados \\
\hline & & Combinación de colores \\
\hline & & Navegación \\
\hline & $\begin{array}{l}\text { Fallas del sistema en } \\
\text { interfaces, } \\
\text { navegación, cálculos } \\
\text { internos, etc. }\end{array}$ & Malos cálculos \\
\hline & & Interfaces de bajo rendimiento \\
\hline & & No amigable \\
\hline
\end{tabular}




\section{PRESUPUESTO}

\begin{tabular}{|l|c|c|}
\hline Rubro de gasto & Parcial, U\$ & Total, U\$ \\
\hline Papelería & 20.00 & 20.00 \\
\hline Tiempo máquina, 200 horas (1 U\$ por hora) & 200.00 & 220.00 \\
\hline $\begin{array}{l}\text { Tiempo análisis y correcciones de diseño, según } \\
\text { bitácora, 300 horas (10 U\$ por hora) }\end{array}$ & $3,000.00$ & $3,220.00$ \\
\hline Total & & $3,220.00$ \\
\hline
\end{tabular}

\section{CRONOGRAMA DE ACTIVIDADES}

\begin{tabular}{|l|c|}
\hline \multicolumn{1}{|c|}{ Actividad } & Fecha \\
\hline Revisión y mejora de documento & Junio de 2014 \\
\hline $\begin{array}{l}\text { Exposición y entrega de protocolo de } \\
\text { investigación }\end{array}$ & 22 de junio de 2014 \\
\hline Aplicación de instrumentos & De agosto de 2014 a septiembre de 2014 \\
\hline Análisis e interpretación de resultados & De octubre de 2014 a noviembre de 2014 \\
\hline Conclusiones & Noviembre de 2014 \\
\hline Recomendaciones & Noviembre de 2014 \\
\hline Anexos y bibliografía & Noviembre de 2014 \\
\hline Revisión y mejora de documento & Noviembre de 2014 \\
\hline
\end{tabular}

\section{REFERENCIA}

Abud Figueroa, M. A. (2001). Calidad en la Industria del Software. La Norma ISO-9126.

Recuperado el 13 de Mayo de 2014, de Universidad Tecnológica de Pereira:

http://recursosbiblioteca.utp.edu.co/tesisd/textoyanexos/0053L864e_anexo.pdf

Allsoft Software Engineering. (Noviembre de 2008). El Modelo CMMI. Recuperado el 7 de Mayo de 2014, de Allsoft: Recursos: http://www.allsoft.com.mx/recursos/ElModeloCMMI.pdf

Broadsword Solutions Corporation. (2014). What is the Capability Maturity Model Integration (CMMI)? Recuperado el 15 de Mayo de 2014, de What is CMMI? | Broadswordsolutions: http://www.broadswordsolutions.com/what-is-cmmi/

Carvallo, J., Franch, X., \& Quer, C. (2009). CALIDAD DE COMPONENTES SOFTWARE. Recuperado el 6 de Mayo de 2014, de Universitat Politècnica de Catalunya: Departament d'Enginyeria de Serveis i Sistemes d'Informació. ESSI.:

http://www.essi.upc.edu/ franch/papers/libro-calidad-cap-10-jpc-xf-cq-10-versionpreliminar.pdf 
Espelt, C. (Junio de 1998). Improving subject retrieval: user-friendly interfaces and effectiveness. Recuperado el 13 de Mayo de 2014, de Universitat de Barcelona: Escola Universitària de Biblioteconomia i Documentació: http://bid.ub.edu/01espel1.htm

Figueroa, F. (25 de Noviembre de 2009). NORMA ISO 9126. Recuperado el 11 de Mayo de 2014, de Blogspot: http://normaiso9126.blogspot.com/

Glazer, H., Dalton, J., Anderson, D., Konrad, M., \& Shrum, S. (Noviembre de 2008). CMMI® or Agile: Why Not Embrace Both! Recuperado el 8 de Mayo de 2014, de Advanced Development Methods, Inc: http://www.controlchaos.com/storage/scrum-articles/CMMi.pdf

Gracia, J. (14 de Agosto de 2005). CMM - CMMI. Recuperado el 9 de Mayo de 2014, de IngenieroSoftware: http://www.ingenierosoftware.com/calidad/cmm-cmmi.php

Johnson, F., Griffiths, J., \& Hartley, R. (Julio de 2003). Task dimensions of user evaluations of information retrieval systems. Recuperado el 12 de Mayo de 2014, de Information Research: http://www.informationr.net/ir/8-4/paper157.html

Nielsen, J. (1 de Enero de 1995a). 10 Usability Heuristics for User Interface Design. Recuperado el 6 de Mayo de 2014, de Nielsen Norman Group: http://www.nngroup.com/articles/tenusability-heuristics/

Nielsen, J. (1 de Enero de 1995b). How to Conduct a Heuristic Evaluation. Recuperado el 14 de Mayo de 2014, de Nielsen Norman Group: http://www.nngroup.com/articles/how-toconduct-a-heuristic-evaluation/

Riol, E. (2010). CMMI para principiantes. Recuperado el 9 de Mayo de 2014, de Eduardo Riol: Ingeniero en Informática: http://www.eduardoriol.com/cmmi-para-principiantes-i/

Tognazzini, B. (5 de Marzo de 2014). First Principles of Interaction Design (Revised \& Expanded). Recuperado el 4 de Mayo de 2014, de AskTOG: Interaction Design Solutions for the Real World: http://asktog.com/atc/principles-of-interaction-design/tutorialspoint. (2014). CMMI Tutorial. Recuperado el 1 de Mayo de 2014, de tutorialspoint: Simply Easy Learning: http://www.tutorialspoint.com/cmmi/index.htm 